\title{
Hydrodynamic emission of strange and non-strange particles at RHIC and LHC
}

\author{
Ulrich Heinz $\uparrow$ \\ † Physics Department, The Ohio State University, Columbus, OH 43210
}

\begin{abstract}
The hydrodynamic model is used to describe the single-particle spectra and elliptic flow of hadrons at RHIC and to predict the emission angle dependence of HBT correlations at RHIC and LHC energies.
\end{abstract}

\section{Introduction and Summary}

One of the key signatures of the formation of thermalized hot and dense matter, such as a quark-gluon plasma (QGP), in relativistic heavy-ion collisions is collective transverse flow, driven by the transverse pressure gradients between the center of the hot collision fireball and the surrounding vacuum. The space-time evolution of this collective flow can be described by relativistic hydrodynamics. In the limit of zero mean free path (i.e. when microscopic scattering rates exceed any macroscopically relevant time scale) the system can be described as an ideal fluid neglecting transport effects such as viscosity and heat conduction. The flow observed in the final state via analysis of the single-particle spectra of emitted hadrons can then be directly related to the initial conditions of the collision fireball when it first thermalized, and to the equation of state $p\left(e, n_{B}\right)$ of the expanding matter.

In non-central collisions the initial conditions are anisotropic, due to the spatial deformation of the nuclear overlap region in the transverse plane, resulting in anisotropic pressure gradients which ultimately lead to anisotropies in the final momentum distributions. The momentum anisotropies can be quantified by the coefficients $v_{n}$ of an azimuthal Fourier decomposition of the transverse momentum distributions. These coefficients, in particular the elliptic flow coefficient $v_{2}$, grow only as long as anisotropies in the spatial pressure gradients persist. Since the reaction zone quickly evolves from its initial spatially deformed state towards a more cylindrical shape without significant anisotropies, the growth of $v_{2}$ is restricted to the earliest stages of the fireball expansion [1]. Even in the absence of interactions among the fireball constituents the spatial anisotropy which could drive the build-up of elliptic flow decays quickly, due to radial free-streaming of the matter [2]. Delayed thermalization therefore manifests itself in reduced elliptic flow since $v_{2}$ then has to build on smaller anisotropies in the pressure gradients. If thermalization happens quickly, elliptic flow builds up earlier and reaches larger values. The resulting stronger flow into the reaction plane [3] destroys the spatial deformation even faster than freestreaming, resulting in an early saturation of the momentum anisotropy after about 5

$\S$ email: heinz@mps.ohio-state.edu 
$\mathrm{fm} / \mathrm{c}$ in semiperipheral $\mathrm{Au}+\mathrm{Au}$ collisions at RHIC [2]. For a given initial deformation (i.e. a fixed impact parameter) the saturation value of $v_{2}$ has been shown to be a monotonic function of the microscopic scattering cross section [4, reaching a strict upper limit given by ideal fluid hydrodynamics in the limit of zero mean free path. The fact that the elliptic flow measured at RHIC almost exhausts this hydrodynamic limit (for an extensive list of references I refer to the recent review [5]) is at this point the strongest available evidence for early thermalization (at $\tau_{\text {therm }}<1 \mathrm{fm} / c$ [ $[$ ] $)$ and, together with an estimated energy density $>10 \mathrm{GeV} / \mathrm{fm}^{3}$ at this early time [2], strongly suggests the formation of a well-thermalized QGP with significant $(5-7 \mathrm{fm} / c)$ lifetime [6].

We have demonstrated elsewhere [5, 6] that the bulk of the observed hadrons from $\mathrm{Au}+\mathrm{Au}$ collisions at RHIC (i.e. all mesons in the region $p_{\mathrm{T}} \lesssim 1.5-2 \mathrm{GeV} / c$ and all baryons at $p_{\mathrm{T}} \lesssim 2-2.5 \mathrm{GeV} / c$ ) behave hydronymically, i.e. that their single particle spectra and momentum anisotropies in central and semicentral collisions with impact parameter $b \lesssim 7 \mathrm{fm}$ can all be quantitatively reproduced by relativistic ideal fluid hydrodynamics with a common kinetic decoupling temperature. In Section 2 I supplement this finding by pointing out that the same holds true even for the heavy $\Omega$ and $\bar{\Omega}$ hyperons, in contrast to earlier expectations based on their presumed weaker coupling to the expanding pion fluid during the late hadronic expansion stage [7. It has also been noted [6, 8, that the hydrodynamic model does much more poorly in its description of the two-particle Bose-Einstein (Hanbury Brown Twiss or HBT) correlations, by overpredicting the longitudinal and outward HBT radii while underpredicting the sideward radius and its transverse momentum dependence (the "RHIC HBT puzzle"). In Section 3 I show how HBT studies as function of the azimuthal emission angle can constrain the space-time evolution of the spatial deformation in non-central collisions, thereby supplementing the information from the measured momentum-space anisotropies and constraining the total duration of the expansion phase. Preliminary RHIC data from 2-pion correlations 9, 10, agree with the hydrodynamic prediction [11] that at kinetic freeze-out the fireball is (on average) still slightly out-of-plane elongated, although much less so than initially. This is expected to change at LHC energies where the much higher initial energy density allows the elliptic flow to act for a significantly longer time period until decoupling, leading to pion emission from an (on average) in-plane elongated source. The HBT signatures of such an in-plane elongation are discussed in Section 3.

\section{Radial flow of $\Omega$ hyperons}

When shortly after the beginning of the experimental program at RHIC in 2000 the first data on the total charged particle multiplicity in central $\mathrm{Au}+\mathrm{Au}$ became available [12], Peter Kolb, Pasi Huovinen and I used this to constrain the initial conditions for the hydrodynamic model in central conditions and to predict hadron spectra and their anisotropies for non-central collisions [13]. Some of these predictions are shown in Fig. [1 The three sets of curves correspond, from top to bottom, to central $(b<5.4 \mathrm{fm})$, semiperipheral $(5.4 \mathrm{fm}<b<9.9 \mathrm{fm})$ and peripheral $(9.9 \mathrm{fm}<b<13.5 \mathrm{fm})$ $\mathrm{Au}+\mathrm{Au}$ collisions at $\sqrt{s}=130 \mathrm{AGeV}$. At that time no spectra were available yet to fix the freeze-out temperature, so we estimated it to probably lie between 120 and 140 $\mathrm{MeV}$ (solid and dashed curves, respectively). We later learned [6] that $T_{\mathrm{f}}=130 \mathrm{MeV}$ fits the pion and proton data from central collisions best. For the $\Omega$ hyperons in the lower right panel we also included a prediction for freeze-out directly after 
hadronization at $T_{\mathrm{c}}=165 \mathrm{MeV}$, motivated by the prevailing prejudice [7] that, due to their lack of resonant scattering with pions, they would not be able to efficiently pick up any additional transverse flow generated in the hadronic phase. This expectation

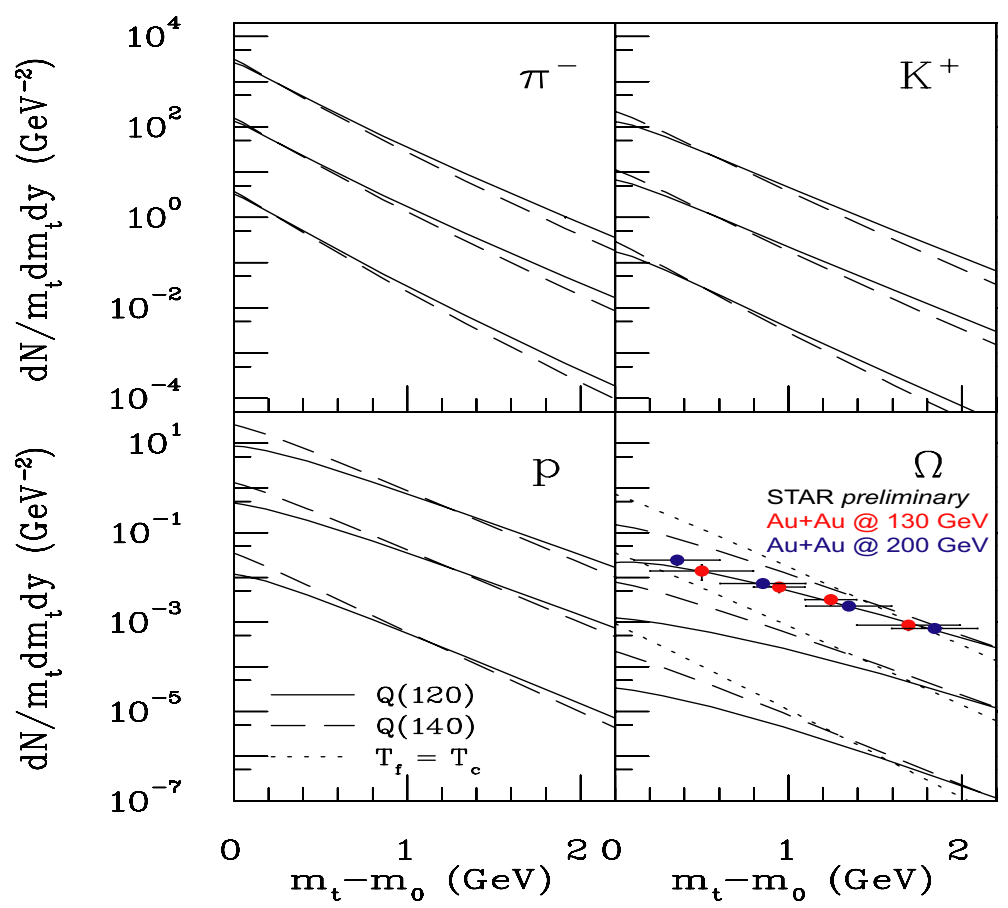

Figure 1. Hydrodynamically predicted hadron spectra from Ref. 13, together with preliminary $\Omega+\bar{\Omega}$ spectra from the STAR collaboration [14. See text for details.

was supported by observations by the WA97 Collaboration at the SPS [15] which indicated a smaller slope parameter for $\Omega$ and $\bar{\Omega}$ than for other hadrons. It was only recently realized that this evidence was at least partially distorted by fitting a curved $\Omega$ spectrum in a limited $m_{\mathrm{T}}$ region by a simple exponential. Recent NA49 data for $\Omega$ and $\bar{\Omega}$ at smaller $m_{\mathrm{T}}$ show evidence that the spectra bend over at low $m_{\mathrm{T}}$ and seem to be consistent with much larger radial flow than previously thought, almost as strong as that experienced by pions and nucleons [16.

Figure 1 shows that the same is true at RHIC: The $\Omega$ spectra from $\mathrm{Au}+\mathrm{Au}$ collisions at $\sqrt{s}=130$ and $200 \mathrm{AGeV} 14$ are consistent with the hydrodynamic predictions if they are assumed to freeze out at the same decoupling temperature $T_{\mathrm{f}} \simeq 130 \mathrm{MeV}$ as pions, kaons and (anti)protons, while they are much too flat to be compatible with freeze-out already at $T_{\mathrm{c}}$, i.e. directly after hadronization. It is impossible to generate enough radial flow for the $\Omega$ s already before hadronization, without destroying the agreement of the model with all other hadron spectra and/or lowering the hadronization temperature significantly below $165 \mathrm{MeV}$ (which would contradict lattice QCD results). We conclude that the $\Omega$ hyperons seem to be much more strongly coupled to the expanding pion fluid during the late hadronic stage than previously thought. It will be interesting to explore the microscopic mechanisms responsible for such a strong coupling. 


\section{Emission angle dependent HBT interferometry}

The observational constraints on dynamical models for the evolution of the heavy-ion reaction zone can be further tightened by a simultaneous analysis of the single-particle momentum spectra with 2-particle momentum correlations. The latter provide access to the space-time structure of the source at kinetic freeze-out. More specifically, in the case of Bose-Einstein correlations between identical bosons, the Gaussian widths $R_{i, j}^{2}(\boldsymbol{K})$ of the correlation function $C(\boldsymbol{q}, \boldsymbol{K})=1+\lambda(\boldsymbol{K}) \exp \left[-\sum_{i, j=o, s, l} q_{i} q_{j} R_{i, j}^{2}(\boldsymbol{K})\right]$ are linear combinations of the components of the spatial correlation tensor $S_{\mu \nu}(\boldsymbol{K})=$ $\left[\left\langle x_{\mu} x_{\nu}\right\rangle-\left\langle x_{\mu}\right\rangle\left\langle x_{\nu}\right\rangle\right](\boldsymbol{K})$ which characterize the space-time widths of the source emission function $S(x, K)$ at freeze-out. For the general case these relations can be found in 17, 18; ; for longitudinally boost-invariant sources and pions at midrapidity $Y=0$ they simplify to

$$
\begin{aligned}
R_{s}^{2}(\Phi)= & \frac{1}{2}\left(S_{x x}+S_{y y}\right)-\frac{1}{2}\left(S_{x x}-S_{y y}\right) \cos 2 \Phi-S_{x y} \sin 2 \Phi \\
R_{o}^{2}(\Phi)= & \frac{1}{2}\left(S_{x x}+S_{y y}\right)+\frac{1}{2}\left(S_{x x}-S_{y y}\right) \cos 2 \Phi+S_{x y} \sin 2 \Phi \\
& -2 \beta_{\mathrm{T}}\left(S_{t x} \cos \Phi+S_{t y} \sin \Phi\right)+\beta_{\mathrm{T}}^{2} S_{t t} \\
R_{o s}^{2}(\Phi)= & S_{x y} \cos 2 \Phi-\frac{1}{2}\left(S_{x x}-S_{y y}\right) \sin 2 \Phi \\
& +\beta_{\mathrm{T}}\left(S_{t x} \sin 2 \Phi-S_{t y} \cos \Phi\right) \\
R_{l}^{2}(\Phi)= & S_{z z} .
\end{aligned}
$$

For simplicity the dependence of the spatial correlation tensor $S_{\mu \nu}$ on the pair momentum $\boldsymbol{K}$ (in particular its implicit dependence on the azimuthal emission angle $\Phi$ of $\boldsymbol{K}$ relative to the reaction plane) has been dropped, and only the explicit $\Phi$-dependence arising from the azimuthal rotation between the outward direction $x_{\text {out }} \| \boldsymbol{K}_{\mathrm{T}}$ and the reaction plane coordinate $x \| \boldsymbol{b}$ is exhibited. For small transverse pair momenta $K_{\mathrm{T}}$ this explicit $\Phi$-dependence dominates [19. The source widths $S_{\mu \nu}$ are specified in coordinates $(x, y)$ parallel and perpendicular to the reaction plane while the correlation function is measured in $\left(x_{\text {out }}, x_{\text {side }}\right)$ coordinates defined by the directions parallel and perpendicular to $\boldsymbol{K}_{\mathrm{T}} . \beta_{\mathrm{T}}$ is the transverse pair velocity. The terms $R_{o l}^{2}$ and $R_{s l}^{2}$ vanish due to the assumed longitudinal boost-invariance.

The orientation of the reaction plane in the laboratory system can be determined event-by-event from the elliptic flow. The correlation function can thus be measured as a function of the azimuthal emission angle $\Phi$ relative to the reaction plane. For non-central collisions the spatial deformation of the source in the transverse plane leads to oscillations as a function of $\Phi$. In practice the finite angular bin size and reaction plane resolution smear these azimuthal oscillations and reduce their measured amplitudes, but this can be fully corrected for in a model-independent way [18. General symmetries ensure that the HBT radii extracted from a Gaussian fit to the thus corrected correlation function exhibit the following general $\Phi$-dependences [18]:

$$
\begin{aligned}
& R_{s}^{2}(\Phi)=R_{s, 0}^{2}+2 \sum_{n=2,4,6, \ldots} R_{s, n}^{2} \cos (n \Phi), \\
& R_{o}^{2}(\Phi)=R_{o, 0}^{2}+2 \sum_{n=2,4,6, \ldots} R_{o, n}^{2} \cos (n \Phi), \\
& R_{o s}^{2}(\Phi)=\quad 2 \sum_{n=2,4,6, \ldots} R_{o s, n}^{2} \sin (n \Phi), \\
& R_{l}^{2}(\Phi)=R_{l, 0}^{2}+2 \sum_{n=2,4,6, \ldots} R_{l, n}^{2} \cos (n \Phi) .
\end{aligned}
$$

For sources without longitudinal boost-invariance $R_{o l}^{2}$ and $R_{o l}^{2}$ oscillate around zero with cosines and sines, respectively, of odd multiples of $\Phi$ [18] 19]. The relations (5) 
continue to hold true for correlation measurements made in a finite symmetric window around $Y=0$.
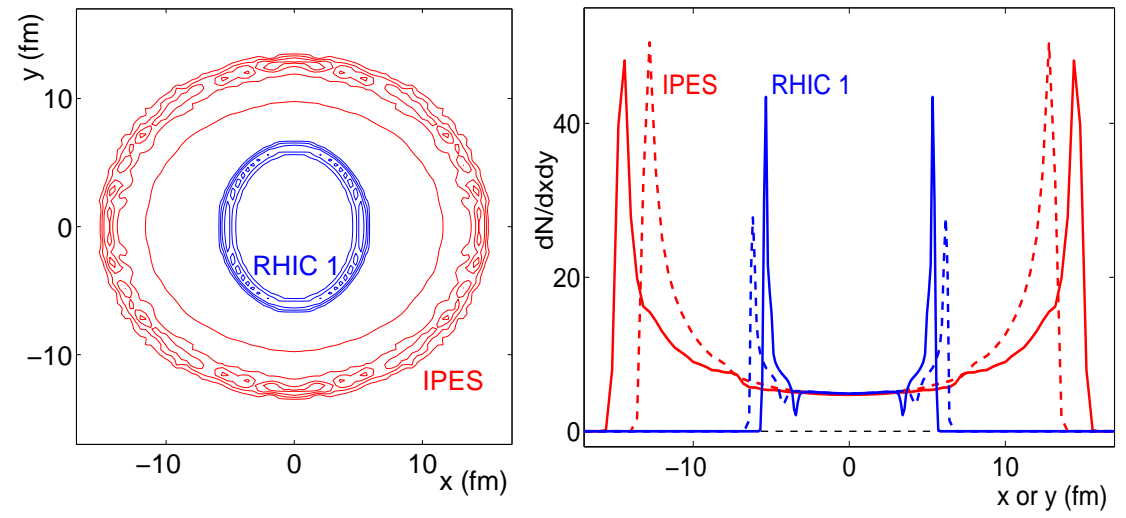

Figure 2. Contour plots of and horizontal (solid) and vertical (dashed) cuts through the $\boldsymbol{K}$-integrated pion emission function in the transverse plane for RHICand LHC-typical initial conditions, for $\mathrm{Au}+\mathrm{Au}$ collisions at $b=7 \mathrm{fm}$ [1].

Figure 2 shows the momentum-integrated hydrodynamic emission function for RHIC- and LHC-type initial conditions. The curves labelled "RHIC 1" correspond to the same initial conditions which led to a successful description of the single-particle momentum spectra and elliptic flow in $130 \mathrm{AGeV} \mathrm{Au+Au}$ collisions. One sees that, when integrated over all momenta and all emission times, this source is still out-ofplane elongated, although only very slightly. "IPES" labels an in-plane-elongated source obtained by starting the hydrodynamics earlier at a higher initial temperature of $2 \mathrm{GeV}$ [11. In this case the source lives longer, grows much bigger and changes its initial out-of-plane elongation into a significant in-plane elongation at freeze-out.

The vertical and horizontal cuts in the right panel of Fig. 2] show that both sources are very "opaque" in that most particles are emitted from the (deformed) surface. A differential analysis shows 11] that only pions with small transverse momenta come from the interior of the source; they are thus the only ones which probe the overall spatial deformation of the source in the transverse plane. Pions with non-zero transverse momenta, on the other hand, come from thin slivers near the edge of the fireball in the emission direction (for details see [11]); they do not directly probe the overall shape of the source.

Figure 3 shows the azimuthal oscillations of the four non-vanishing HBT radius parameters for several values of $K_{\mathrm{T}}$, calculated from these emission functions. The opposite spatial deformation of the RHIC 1 and IPES sources is reflected in the opposite signs of the oscillations for $R_{s}^{2}, R_{o}^{2}$ and $R_{o s}^{2}$ at $K_{\mathrm{T}}=0$. For example, at RHIC energies $R_{s}^{2}$ oscillates downward, implying a larger sideward radius when viewed from the $x$ direction (i.e. within the reaction plane) than from the $y$-direction (i.e. perpendicular to the reaction plane). For the in-plane elongated source (IPES) some of the oscillation amplitudes change sign at larger transverse momenta. This change of sign originates from an intricate interplay between geometric, dynamical and temporal aspects of the source at freeze-out [11] and reinforces the statement that at $K_{\mathrm{T}} \neq 0$ the HBT correlations do not probe the entire source and its spatial shape.

Preliminary STAR data on azimuthal oscillations of the HBT radii 9 10 confirm the oscillation pattern in the left panel of Fig. 3 but disagree with the one shown in 

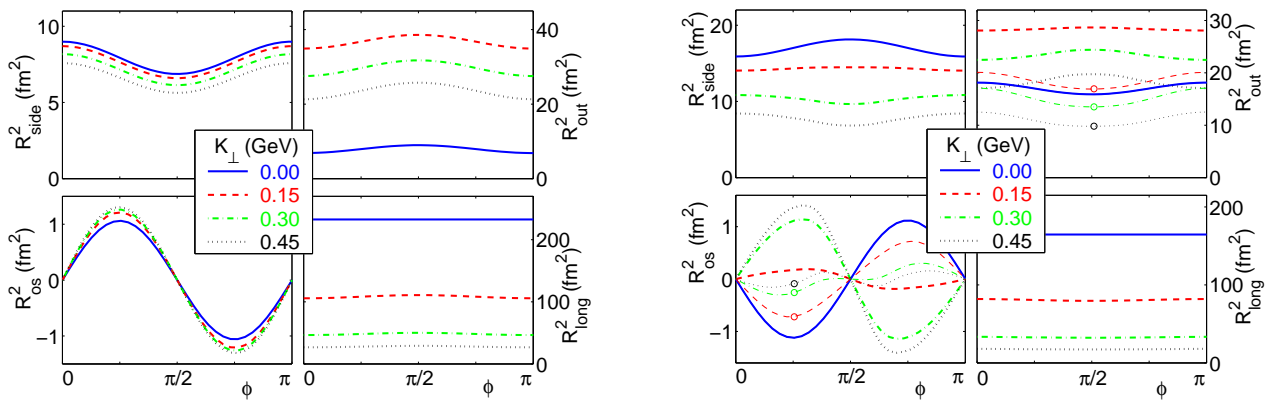

Figure 3. Oscillations of the HBT radii for different transverse pair-momenta in RHIC collisions (left) and for the source elongated into the reaction plane (right) 11. The thin circled lines in the right panel show the geometric contributions to the HBT radius parameters.

the right panel. This suggests that hydrodynamics gets the overall duration of the expansion stage about right, since significantly longer times until freeze-out should cause the source to reverse the sign of its spatial deformation. More quantitative statements have to await a resolution of the above-mentioned "HBT puzzle" in central collisions.

The significance of the $K_{\mathrm{T}}$-dependence of the oscillation amplitudes for the HBT radius parameters is still largely unexplored. From the analysis of central collisions 20] it is known that the $\Phi$-averaged transverse HBT radii (in particular $R_{o}$ ) exhibit stronger $K_{\mathrm{T}}$ dependence for sources with a sharp surface (such as the hydrodynamic one obtained from the Cooper-Frye prescription) than for Gaussian sources. One might expect similarly strong differences for the $K_{\mathrm{T}}$-dependence of the oscillation amplitudes in non-central collisions.

Acknowledgement: This work was supported by the U.S. Department of Energy under Grant No. DE-FG02-01ER41190.

\section{References}

[1] Sorge S 1997 Phys. Rev. Lett. 782309.

[2] Kolb P F, Sollfrank J and Heinz U 2000 Phys. Rev. C 62054909.

[3] Ollitrault J-Y 1992 Phys. Rev. D 46229.

[4] Zhang B, Gyulassy M and Ko C M 1999 Phys. Lett. B 45545.

[5] Kolb P F and Heinz U 2003 Preprint arXiv:nucl-th/0305084.

[6] Heinz U and Kolb P F 2002 Nucl. Phys. A 702269.

[7] van Hecke H, Sorge H and Xu N 1998 Phys. Rev. Lett. 815784.

[8] Heinz U and Kolb P F 2002 Preprint arXiv:nucl-th/0305084, Proceedings of the 18th Winter Workshop on Nuclear Dynamics, Nassau, Bahamas, 20-27, 2002, (G.D. Westfall and W. Bauer, eds.), EP Systema, Debrecen, Hungary (2002).

[9] López Noriega M et al. (STAR Collaboration) 2003 Nucl. Phys. A 715 623c.

[10] Lisa M A et al. (STAR Collaboration) 2002 Preprint arXiv:nucl-ex/0301005.

[11] Heinz U and Kolb P F 2002 Phys. Lett. B 542216.

[12] Back B B et al. (PHOBOS Collaboration) 2000 Phys. Rev. Lett. 853100.

[13] Huovinen P, Kolb P F, Heinz U, Ruuskanen P V and Voloshin S A, 2001 Phys. Lett. B 50358.

[14] Suire C et al. (STAR Collaboration) 2003 Nucl. Phys. A 715 470c.

[15] Antinori F et al. (WA97 Collaboration) 2000 Eur. Phys. J. C 14633.

[16] van Leeuwen M for the NA49 Collaboration 2003 Nucl. Phys. A $715161 c$.

[17] Wiedemann U A 1998 Phys. Rev. C 57266.

[18] Heinz U, Hummel A, Lisa M A and Wiedemann U A 2002 Phys. Rev. C 66044903.

[19] Lisa M A, Heinz U and Wiedemann U A 2000 Phys. Lett. B 489287.

[20] Tomášik B, Wiedemann U A and Heinz U 2003 Heavy Ion Phys. 17105. 